\title{
Home Security and Surveillance using glass breaking and baby crying detection and Audio Classification
}

\author{
Shivansh Mittal $^{1}$ I Sachin Garg ${ }^{2}$ \\ ${ }^{1}$ UG student, IT \& Maharaja Agrasen Institute Of Technology,Delhi,India \\ ${ }^{2}$ Asst. Prof. Department of IT \& ,Maharaja Agrasen Institute Of Technology,Delhi,India
}

To Cite this Article

Shivansh Mittal and Sachin Garg, "Home Security and Surveillance using glass breaking and baby crying detection and Audio Classification", International Journal for Modern Trends in Science and Technology, 6(12): 459-462, 2020.

\section{Article Info}

Received on 16-November-2020, Revised on 09-December-2020, Accepted on 12-December-2020, Published on 19-December-2020.

\section{ABSTRACT}

The main objective of this project is to detect audio which may be vulnerable to people living in a family environment and ensure security at their homes.

All the process from data collection to data cleaning and then training and testing the finally made Machine Learning model will be done for audio's like Glass breaking and Baby Crying Detection.

This model can be used in smart home devices to ensure safety and security at the homes of people.

KEYWORDS:Glass Breaking Audio Detection, Baby Crying Audio Detection, Audio Classification, Librosa

\section{INTRODUCTION}

This project is based on classification of Audio Samples and detection of audio. We will be first collecting the audio dataset for the project. Then will be cleaning the whole data and splitting it into equi-length samples. This will be followed by feature extraction from the audio samples fitting it into machine learning models and collecting the results providing best accuracies against test samples. The audio detection will be of audio data like Glass breaking, Baby crying, Gun Shot etc. to ensure safety and security in homes.

This project provides an overview to how Audio can be used as a surveillance tool to ensure safety and security at homes. Librosa plays a very vital role in Audio feature extraction in the project and helps in extraction of features like MFCC, Chroma, Spectral Contrast etc.
The project will be able to give a very a through idea about Audio Classification using Machine Learning and improving security in Future Homes.

\section{Overview}

Problem: Problem is to use Audio detection for ensuring security in homes.

Data: The data used here is taken from various sources like YouTube, FreeSound and has been cropped to a particular sample size.

The data taken is for audio classification and detection of audio like Glass breaking, Baby crying, Gun Shot detection etc.

Evaluation: The evaluation would be on the basis of weather the final model is able to detect the audio correctly or not.

Features: Would be using a lot of features to classify audio samples like MFCC (Mel- Frequency 
Spectral Coefficient), Chroma, Spectral Contrast, Mel, Tonnetz etc.

Dataset-

- 1045 glass breaking mono audio samples of 6 seconds at $22.5 \mathrm{kHz}$.

- 1350 baby crying mono audio samples of 6 seconds at $22.5 \mathrm{kHz}$.

- 1700 noise mono audio samples of 6 seconds at $22.5 \mathrm{kHz}$.

\section{Methodology}

The process description of this work include the following:

1.Setting up the data: In this the data is arranged and formatted such that it is compatible for the training usage and there are no missing values or misguiding arrangements.

2. Preparing the Audio data: In this process the Audio samples are now cropped to a particular length such that all the samples are of the same length and frequency.

3. Feature Selection and Fitting the model: Features are selected and fit to the machine learning model and predictions are made from these data sets and accuracies are recorded.

4.Improve the accuracy through experiments: The model accuracy has to be improved by changing the hyper parameters and making random and associated valued to these variables.

5. Save and reload the model for future predictions: The model that is trained is saved or logged in some manner such that the prediction labels can be determined by the trained model.

\section{RESULTS}

Features Extracted- 64 features using MFCC

Parameters Used for MFCC-

- n_fft= 2048

- hop_length= 512

As $n$ fft should be greater than sample length and should be a power of 2 .

Creating Dataset

- Extracted MFCC features from dataset and created '.npy' files for training.

Training Model Used- Support Vector Machine

\section{Features Table}

\begin{tabular}{|l|l|l|l|}
\hline S.No & Features & \multicolumn{1}{|c|}{ Explanation } & \multicolumn{1}{|c|}{ Working } \\
\hline 1. & MFCC & $\begin{array}{l}\text { Graph plotting } \\
\text { shows great } \\
\text { contrast and } \\
\text { less overlapping }\end{array}$ & YES \\
\hline 2. & Mel & $\begin{array}{l}\text { Averaging lead } \\
\text { to bad results }\end{array}$ & NO \\
\hline 3. & Tonnetz & $\begin{array}{l}\text { Overlapping of } \\
\text { Points }\end{array}$ & NO \\
\hline 4. & $\begin{array}{l}\text { Spectral } \\
\text { Contrast }\end{array}$ & $\begin{array}{l}\text { Overlapping of } \\
\text { Points }\end{array}$ & NO \\
\hline 5. & Chroma & $\begin{array}{l}\text { Overlapping of } \\
\text { Points }\end{array}$ & NO \\
\hline
\end{tabular}

\section{Hyperplane Plots of Best Results}

17. Cry+Noise(New)

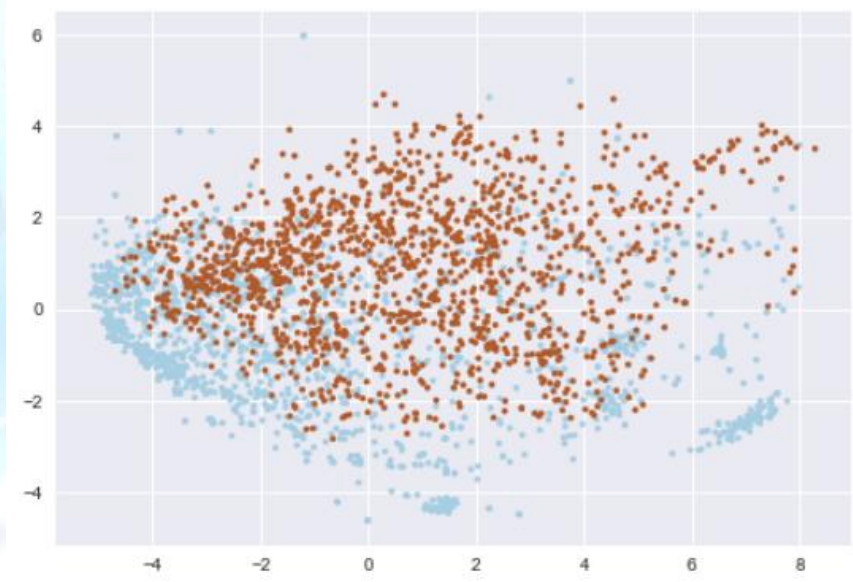

14. Cry+Glass+Noise(New)

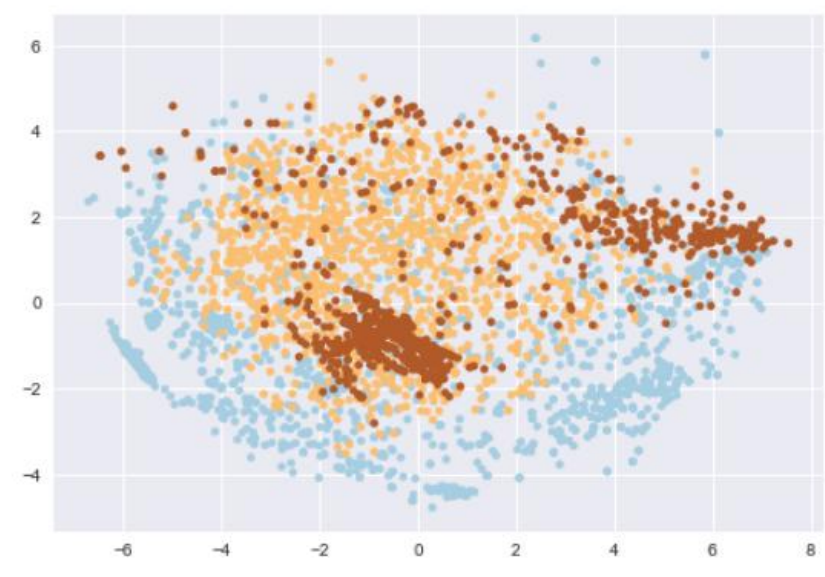


RESULTS TABLE

\begin{tabular}{|c|c|c|c|c|c|c|c|}
\hline S.No & Dataset & Features & Pre-empha & Parameters & & Accuracy & \\
\hline & & & & & Cry & Glass & Noise \\
\hline 1. & Cry+Noise & MFCC & $1 / 16 \mathrm{kHz}$ & $\begin{array}{l}\text { 'C': 10, 'degree': 2, } \\
\text { 'gamma': 0.0001, } \\
\text { 'kernel': 'rbf' }\end{array}$ & $75.7 \%$ & - & $90.6 \%$ \\
\hline 2. & Cry+Glass & MFCC & $1 / 16 \mathrm{kHz}$ & $\begin{array}{l}\text { 'C': 10, 'degree': } 2 \text {, } \\
\text { 'gamma': 0.0001, } \\
\text { 'kernel': 'rbf' }\end{array}$ & & $91.05 \%$ & $100 \%$ \\
\hline 3. & $\begin{array}{l}\text { Cry+Glass+ } \\
\text { Noise }\end{array}$ & MFCC & $1 / 16 \mathrm{kHz}$ & $\begin{array}{l}\text { 'C': 10, 'degree': 2, } \\
\text { 'gamma': } 0.0001 \text {, } \\
\text { 'kernel': 'rbf' }\end{array}$ & $81.4 \%$ & $80 \%$ & $82.6 \%$ \\
\hline 4. & $\begin{array}{l}\text { Cry+Glass+ } \\
\text { Noise }\end{array}$ & MFCC & $5 / 16 \mathrm{kHz}$ & $\begin{array}{l}\text { 'C': 10, 'degree': 2, } \\
\text { 'gamma': 0.0001, } \\
\text { 'kernel': 'rbf' }\end{array}$ & $82.4 \%$ & $80.4 \%$ & $82.7 \%$ \\
\hline 5. & $\begin{array}{l}\text { Cry+Glass+ } \\
\text { Noise }\end{array}$ & MFCC & $10 / 16 \mathrm{kHz}$ & $\begin{array}{l}\text { 'C': 10, 'degree': } 2 \text {, } \\
\text { 'gamma': } 0.0001, \\
\text { 'kernel': 'rbf' }\end{array}$ & $81.9 \%$ & $84.17 \%$ & $80.4 \%$ \\
\hline 6. & $\begin{array}{l}\text { Cry+Glass+ } \\
\text { Noise }\end{array}$ & MFCC & $3 / 16 \mathrm{kHz}$ & $\begin{array}{l}\text { 'C': 10, 'degree': 2, } \\
\text { 'gamma': 0.0001, } \\
\text { 'kernel': 'rbf' }\end{array}$ & $82.4 \%$ & $81.2 \%$ & $80.9 \%$ \\
\hline 7. & $\begin{array}{l}\text { Cry+Glass+ } \\
\text { Noise }\end{array}$ & MFCC & $5 / 16 \mathrm{kHz}$ & $\begin{array}{l}\text { 'C': 10, 'degree': } 2 \text {, } \\
\text { 'gamma': } 0.0001, \\
\text { 'kernel': 'rbf' }\end{array}$ & $80.9 \%$ & $89.2 \%$ & $81.6 \%$ \\
\hline 8. & $\begin{array}{l}\text { Cry+Glass+ } \\
\text { Noise }\end{array}$ & MFCC & $1 / 16 \mathrm{kHz}$ & $\begin{array}{l}\text { 'C': 10, 'degree': } 2 \text {, } \\
\text { 'gamma': } 0.0001, \\
\text { 'kernel': 'rbf' }\end{array}$ & $77.7 \%$ & $84.7 \%$ & $88.3 \%$ \\
\hline $9 . \quad 7$ & $\begin{array}{l}\text { Cry+Glass }+ \\
\text { Noise }\end{array}$ & MFCC & $1 / 22.5 \mathrm{kHz}$ & $\begin{array}{l}\text { 'C': 10, 'degree': } 2 \text {, } \\
\text { 'gamma': } 0.0001, \\
\text { 'kernel': 'rbf' }\end{array}$ & $83.2 \%$ & $82.7 \%$ & $82.01 \%$ \\
\hline 10. & $\begin{array}{l}\text { Cry+Glass+Nois } \\
\text { e }\end{array}$ & MFCC & $5 / 22.5 \mathrm{kHz}$ & $\begin{array}{l}\text { 'C': 15, 'degree': } 2 \text {, } \\
\text { 'gamma': } 0.0001, \\
\text { 'kernel': 'rbf' }\end{array}$ & $86.7 \%$ & $86.7 \%$ & $81.7 \%$ \\
\hline 11. & $\begin{array}{l}\text { Cry+Glass+ } \\
\text { Noise }\end{array}$ & MFCC & $5 / 22.5 \mathrm{kHz}$ & $\begin{array}{l}\text { 'C': 15, 'degree': } 2 \text {, } \\
\text { 'gamma': } 0.0001, \\
\text { 'kernel': 'rbf' }\end{array}$ & $83.5 \%$ & $81.6 \%$ & $80.8 \%$ \\
\hline 12. & $\begin{array}{l}\text { Cry+Glass+Nois } \\
\text { e }\end{array}$ & $\begin{array}{l}\text { MFCC } \\
\text { Normalized-Au } \\
\text { dio }\end{array}$ & $5 / 22.5 \mathrm{kHz}$ & $\begin{array}{l}\text { 'C': 15, 'degree': 2, } \\
\text { 'gamma': } 0.0001, \\
\text { 'kernel': 'rbf' }\end{array}$ & $89.9 \%$ & $75.7 \%$ & $82.7 \%$ \\
\hline 13. & $\begin{array}{l}\text { Cry+Glass+Nois } \\
\mathrm{e}\end{array}$ & $\begin{array}{l}\text { MFCC } \\
\text { Normalized-Au } \\
\text { dio }\end{array}$ & $5 / 22.5 \mathrm{kHz}$ & $\begin{array}{l}\text { 'C': 30, 'degree': 2, } \\
\text { 'gamma': } 0.0001, \\
\text { 'kernel': 'rbf' }\end{array}$ & $91.05 \%$ & $84.7 \%$ & $75.7 \%$ \\
\hline 14. & $\begin{array}{l}\text { Cry+Glass+ } \\
\text { Noise }\end{array}$ & $\begin{array}{l}\text { MFCC } \\
\text { Normalized-Au } \\
\text { dio+Feature }\end{array}$ & $5 / 22.5 \mathrm{kHz}$ & $\begin{array}{l}\text { 'C': } 35, \text { 'degree': } 2 \text {, } \\
\text { 'gamma': } 0.1 \text {, } \\
\text { 'kernel': 'rbf' }\end{array}$ & $93.6 \%$ & $80.6 \%$ & $80.1 \%$ \\
\hline 15. & $\begin{array}{l}\text { Cry+Glass }+ \\
\text { Noise }\end{array}$ & $\begin{array}{l}\text { MFCC } \\
\text { Normalized-Fe } \\
\text { ature }\end{array}$ & $5 / 22.5 \mathrm{kHz}$ & $\begin{array}{l}\text { 'C': 10, 'degree': 2, } \\
\text { 'gamma': } 0.1 \text {, } \\
\text { 'kernel': 'rbf' }\end{array}$ & $93.12 \%$ & $81.6 \%$ & $73.5 \%$ \\
\hline 16. & Cry+Noise & $\begin{array}{l}\text { MFCC } \\
\text { Normalized-Fe } \\
\text { ature+Audio }\end{array}$ & $5 / 22.5 \mathrm{kHz}$ & $\begin{array}{l}\text { 'C': 10, 'degree': } 2 \text {, } \\
\text { 'gamma': } 0.1 \text {, } \\
\text { 'kernel': 'rbf' }\end{array}$ & $90.4 \%$ & - & $78.6 \%$ \\
\hline 17. & Cry+Noise & $\begin{array}{l}\text { MFCC } \\
\text { Normalized-Fe } \\
\text { ature }\end{array}$ & $5 / 22.5 \mathrm{kHz}$ & $\begin{array}{l}\text { 'C': } 5 \text {, 'degree': } 2 \text {, } \\
\text { 'gamma': } 0.1 \text {, } \\
\text { 'kernel': 'rbf' }\end{array}$ & $87.3 \%$ & - & $85.3 \%$ \\
\hline 18. & Cry+Noise & $\begin{array}{l}\text { MFCC } \\
\text { Normalized-Au } \\
\text { dio }\end{array}$ & $5 / 22.5 \mathrm{kHz}$ & $\begin{array}{l}\text { 'C': 10, 'degree': 3, } \\
\text { 'gamma': 0.01, } \\
\text { 'kernel': 'poly' }\end{array}$ & $84.1 \%$ & - & $77.9 \%$ \\
\hline
\end{tabular}




\section{CONCLUSION}

This project provides an overview to how Audio can be used as a surveillance tool to ensure safety and security at homes. Librosa plays a very vital role in Audio feature extraction in the project and helps in extraction of features like MFCC, Chroma, Spectral Contrast etc.

The project will be able to give a very a through idea about Audio Classification using Machine Learning and improving security in Future Homes.

Glass Breaking and Baby crying detection has been done and good accuracies have been found using SVM model with 64 normalized MFCC features with normalized audio.

\section{REFERENCES}

[1] Music Type Classification By Spectral Contrast Feature1 The work was performed at Microsoft Research Asia Dan-Ning Jiang, Lie Lu, Hong-Jiang Zhang, Jian-Hua Tao, Lian-Hong Cai

[2] B. Matityaho and M. Furst. "Neural Network Based Model for Classification of Music Type", in Proc. of 18th Conv. Electrical and Electronic Engineers in Israel, pp. 4.3.4/1-5, 1995.

[3] K. P. Han, Y. S. Pank, S. G. Jeon, G.C. Lee, and Y. H. Ha, "Genre Classification System of TV Sound Signals Based on a Spectrogram Analysis", IEEE Transactions on Consumer Electronics, VOL. 55, No. 1, pp. 33-42, 1998.

[4] H. Soltau, T. Schultz, Martin Westphal, and Alex Waibel. "Recognition of Music Types". ICASSP 1998, Vol. II, pp. 1137-1140, 1998.

[5] D. Pye. "Content-Based Methods for the Management ofDigital Music". ICASSP 2000, Vol. IV, pp.2437-2440, 2000.

[6] D. Li, I. K. Sethi, N. Dimitrova, T. McGee, "Classificationof General Audio Data for Content-BasedRetrieval", PatternRecognition Letters, VOL. 22, No. 5, pp. 533-544, 2001.

[7] S. Sadie as Editor, "The Cambridge Music Guide",Cambridge University Press, 1985.

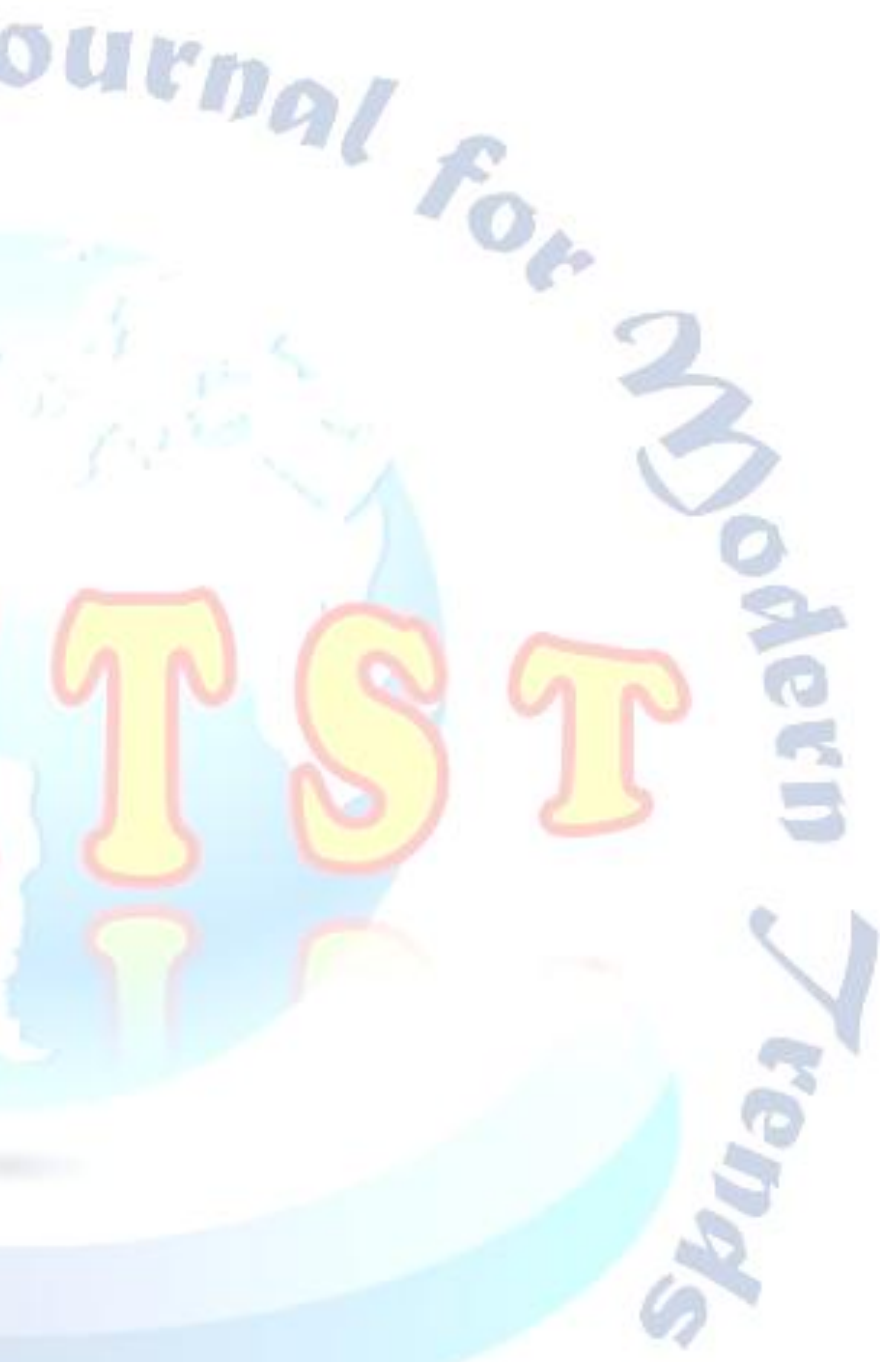

